International Journal of Energy and Statistics

Vol. 3, No. 4 (2015) 1599001 (2 pages)

(C) Institute for International Energy Studies

DOI: $10.1142 / \mathrm{S} 233568041599001 \mathrm{X}$

\title{
Author index (Volume 3)
}

Ahmadi, S., see Fakehi,

A. $\mathrm{H}$.

1 (2015) 1550004

Alkhaldi, R., see

Al-Mashhadani, A.

Al-Mashhadani, A.,

Elseed, Md. G.,

Alkhaldi, R.,

Al-Sachit, S. \&

Al-Washahi, W.,

Optimization of $U K$

generation mix for

2030

Al-Sachit, S., see

Al-Mashhadani, A.

Al-Washahi, W., see

Al-Mashhadani, A.

Amipara, D. V., see

Engineer, H. R.

Bagheri, M. A., see

Moosavi, A.

Cyrino Oliveira, F. L., see Maçaira, P. M.

Dehnavi, J., Wirl, F. \&

Yegorov, Y.,

Arbitrage in natural

gas markets?

Deris, M. M., see

Efendi, R.

Destek, M. A. \& Ozsoy,

F. N., Relationships

between economic

growth, energy

consumption,

globalization,

urbanization and

environmental

degradation in

Turkey

Efendi, R., Ismail, Z.,

Sarmin, N. H. \&

Deris, M. M., A

reversal model of

3 (2015) 1550011

3 (2015) 1550011

3 (2015) 1550011

3 (2015) 1550011

4 (2015) 1550019

2 (2015) 1550008

1 (2015) 1550005

1 (2015) 1550003
4 (2015) 1550017 fuzzy time series in

regional load

forecasting

1 (2015) 1550003

Elseed, Md. G., see

Al-Mashhadani, A.

Engineer, H. R.,

Amipara, D. V.,

Kacha, D. R.,

Vadgama, D. A. \&

Patel, A. M., A

review on the

potential sources for producing electricity with thermoelectric generators and different configurations

Fakehi, A. H., Ahmadi, S., Ghofrani, Md. B. \& Saboohi, Y., A multi-regional model for power generation expansion planning

Fianu, E. S., Portfolio optimization in zonal energy markets: Evidence

from Italy

Franco, E. M. C., see Júnior, L. A. T. 2 (2015) 1550006

2 (2015) 1550007

Ghodsi, M. \& Huang,

X., Causality between energy poverty and economic growth in Africa: Evidences from time and frequency domain causality test

4 (2015) 1550020

Ghodsi, M., Trend extraction in world oil prices
4 (2015) 1550019

1 (2015) 1550004 
Ghodsi, Z., The impact of disaggregation on the forecasting performance of natural gas price

Ghofrani, Md. B., see Fakehi, A. H.

Huang, X., see Ghodsi, M.

Huang, X., A comparison of association measures: Evidence from stock markets and oil prices

Ismail, Z., see Efendi, $\mathrm{R}$.

Júnior, L. A. T., Rodrigues, S. B., Franco, E. M. C. \& Lindbeck da Silva,

A. C., A wavelet hybrid method based on ARIMA and support vector regression forecasts

Kacha, D. R., see Engineer, H. R.

Khasteh, S. H., see Moosavi, A.

Li, J. \& Zheng, B., Digital core and pore network model reconstruction based on random fractal theory

Lindbeck da Silva, A. C., see Júnior, L. A. $\mathrm{T}$.

Liu, S., see Zhao, X.

Lorestani, H., see Sayyareh, A.

Maçaira, P. M., Cyrino Oliveira, F. L. \& Souza, R. C., Forecasting natural inflow energy series with multi-channel singular spectrum analysis and bootstrap techniques

Moosavi, A., Khasteh, S. H. \& Bagheri, M. A., Forecasting crude oil price with ensemble neural networks based on different feature subsets method

Ozsoy, F. N., see Destek, M. A.

Patel, A. M., see Engineer, H. R.

Rodrigues, S. B., see Júnior, L. A. T.

2 (2015) 1550008

1 (2015) 1550004

4 (2015) 1550020

3 (2015) 1550012

1 (2015) 1550003

Saboohi, Y., see Fakehi, A. H.

Sarmin, N. H., see Efendi, R.

Sayyareh, A. \& Lorestani, H., Statistical model for electrical energy production

4 (2015) 1550016

Silva, E. S., A short note on the accuracy of structural break tests at detecting oil price shocks in advance

4 (2015) 1550017

4 (2015) 1550019

2 (2015) 1550007

1 (2015) 1550004

1 (2015) 1550003

Souza, R. C., see Maçaira, P. M.

Vadgama, D. A., see Engineer, H. R.

Wang, B., see Zhao, X.

Wirl, F., see Dehnavi, J.

4 (2015) 1550018

Yang, D., Simulation study of parameter estimation and measurement planning on photovoltaics degradation

3 (2015) 1550013

1 (2015) 1550002

4 (2015) 1550016

Yarmohammadi, M., Predicting oil prices with the forecast package in $R$

Yegorov, Y., see Dehnavi, J.

2 (2015) 1550009

1 (2015) 1550005

4 (2015) 1550019

1 (2015) 1550002

Zhao, X., Wang, B. \& Liu, S., Assessing the level of low carbon development for developing countries $\quad \mathbf{1}$ (2015) 1550002 Zheng, B., see Li, J. $\quad 1$ (2015) 1550001 